Journal of Case Reports 2017;7(1):68-70

\title{
Infective Endocarditis: A Decade after Ross Procedure
}

\author{
OS Suman ${ }^{1}$, MV Teena ${ }^{1}$, AR Muneer ${ }^{1}$, N Ramesh ${ }^{1}$, G Vijayaraghavan ${ }^{1}$, AN Kalyagin² \\ ${ }^{1}$ Department of Cardiology, Kerala Institute of Medical Sciences, Trivandrum, India; ${ }^{2}$ Department of Medicine, Irkutsk State \\ Medical University, Irkutsk, Russia.
}

\author{
Corresponding Author: \\ Dr. OS Suman \\ Email: suman_os@yahoo.com \\ This is an Open Access article distributed \\ under the terms of the Creative Commons \\ Attribution License (creativecommons.org/ \\ licenses/by/3.0). \\ Received : November 20, 2016 \\ Accepted : January 23, 2017 \\ Published : February 20, 2017
}

\begin{abstract}
Background: The Ross procedure is an acceptable alternative to conventional aortic valve replacement in selected patients. The Ross procedure was found to have a lower incidence of infective endocarditis during follow-up compared to other traditional aortic replacement procedure. Case Report: We hereby report a 54 year gentleman with infective endocarditis, 10 years after the Ross procedure. Conclusion: Conservative six weeks antibiotic treatment is effective for management of pulmonary homograft endocarditis.
\end{abstract}

Keywords: Allograft, Autograft, Aortic valve, Endocarditis, Heart Valve Prosthesis.

\section{Introduction}

The Ross procedure is named after Dr. Donald Ross - a pioneer in cardiac surgery from UK who proposed the procedure in 1962 [1] and first performed it in 1967. In Ross procedure diseased aortic valve is replaced with the patient's own pulmonary valve. A pulmonary allograft (valve taken from cadaver) is then used to replace the patient's pulmonary valve. Pulmonary autograft replacement of the aortic valve is the operation of choice in infants and children, but its use in adults remains controversial [2]. Advantages of this therapeutic option are the use of the patients' own valve with favourable hemodynamic characteristics, low thrombogenicity, and the potential to grow in children. We are reporting a case of infective endocarditis 10 years after a Ross procedure.

\section{Case Report}

A 53 year old gentleman presented with intermittent fever since last 6 months. He had lost 8 kilograms of weight and felt extremely tired. He had several courses of antibiotics in different hospitals. He underwent balloon angioplasty for coarctation of aorta when he was 37 years old. He underwent pulmonary autograft replacement of aortic valve with homograft replacement of pulmonary valve for aortic stenosis when he was 42 years old. For a non-ST elevation myocardial infarction, left anterior descending coronary angioplasty was done when he was 48 years old.

Clinical examination revealed a heart rate of $72 / \mathrm{min}$, normal blood pressure and there was no cardiomegaly. A 3/6 ejection systolic murmur was noted in the left sternal border. The electrocardiogram showed sinus rhythm with rate $72 /$ min. Chest X-ray showed no cardiomegaly and sputum AFB was negative. The CT chest showed a patchy fibrosis of the right lower zone with nodular opacities with central clearing [Fig.1]. The echo Doppler studies revealed normal cardiac chambers, right ventricular outflow tract and pulmonary homograft was not clearly visualized due to poor acoustic window. The colour Doppler showed turbulence across the pulmonary valve and a systolic gradient of $60 \mathrm{mmHg}$ was noted. The transesophageal echocardiogram delineated the 
right ventricular outflow tract with the pulmonary valve showing multiple mobile pedunculated freely mobile masses attached to the homograft leaflets [Fig.2]. The blood culture grew Staphylococcus haemolyticus which was sensitive to linezolid. The patient was treated with intravenous linezolid for 6 weeks. He became afebrile and repeated blood cultures were negative. Transesophageal echocardiogram repeated after 6 weeks showed reduction in the size and number of vegetations [Fig.3] and the gradient in the right ventricle to pulmonary artery was reduced to $34 \mathrm{mmHg}$.

\section{Discussion}

Yearsafterthe Rossprocedure, infectiveendocarditis has been very rare. Asrafian et al. reported a case of pulmonary homograft endocarditis 19 years after Ross procedure, secondary to Streptococcus agalactiae from a cat bite [3]. The patient underwent successful pulmonary vegetectomy and homograft replacement. Another case of endocarditis after the Ross procedure was reported by Gamez A et al. and they reported that Ross procedure is presumed to have a lower incidence of infective endocarditis during follow-up compared to other aortic replacement procedures [4]. They describe four cases of infective endocarditis during follow-up of 96 patients who underwent the Ross procedure. One patient required surgery during the active phase of the infective endocarditis. The clinical outcome was good in all patients and no patient died during follow-up.

The Ross operation is perhaps the most ideal alternative to prosthetic aortic valve replacement in patients suffering from a variety of aortic valve diseases. Both the Ross procedure and mechanical prosthesis implantation have important advantages and disadvantages. Because of the increased thrombogenicity of mechanical prostheses, the choice for this valve substitute implies lifelong anticoagulation and is associated with an increased risk for thromboembolic and bleeding events. The use of anticoagulation may also complicate pregnancy because of the fetal

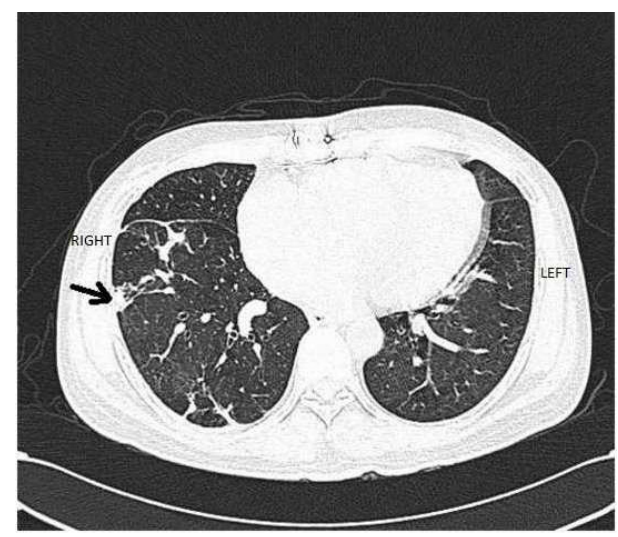

Fig.1: CT scan shows patchy fibrosis of the lower zone of right lung.

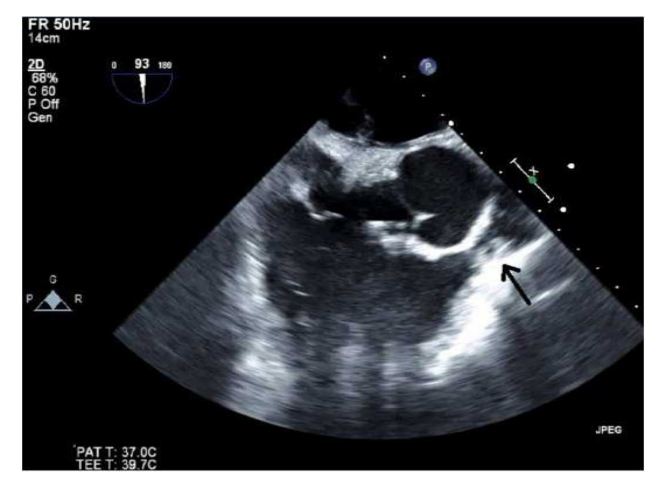

Fig.2: Transesophageal echocardiogram shows multiple vegetations in the pulmonary homograft.

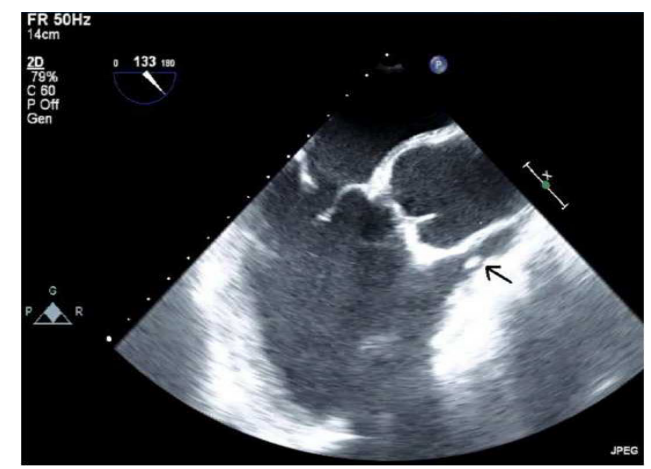

Fig.3: Transesophageal echocardiogram shows reduction in the size of vegetat.

and maternal complications of taking warfarin $[5,6]$ and may require lifestyle adjustments in this relatively young and active patient group. Furthermore, compared with autograft valves, the 
hemodynamic performance of mechanical valves is less favourable, and mechanical valve noise can negatively affect the patient's quality of life [7]. The choice for a Ross procedure on the other hand, would mean a limited durability of the aortic valve autograft and pulmonary valve allograft and implies a certain risk of reoperation during the patient's life, depending on the technique used and the follow-up time.

The advantages of the Ross procedure are the superior hemodynamic performance, low valverelated event occurrence rates, and no need for lifelong anticoagulation. Infection of the pulmonary homograft is extremely rare and the difficulty in diagnosis is being highlighted. In our patient, we could manage the infection conservatively. Blood culture and transesophageal echocardiogram helped to confirm the diagnosis. Remarkable recovery of specific antibiotic treatment prompted to re-evaluate the patient regarding reintervention. Within two weeks of medical treatment the gradient reduced by $42 \mathrm{mmHg}$ and it was decided to proceed with conservative management. After six weeks of treatment with antibiotics the gradient reduced to $34 \mathrm{mmHg}$ and the patient recovered well. Our patient had received multiple short courses of antibiotics from different hospitals outside. Hence the diagnosis was delayed for almost 6 months being a case of partially treated infective endocarditis .Since the organism responsible was Staphylococcus haemolyticus, the course of the disease was rather indolent.

\section{Conclusion}

Infective endocarditis is rarely reported after Ross procedure. We report a case of pulmonary homograft endocarditis 10 years after Ross procedure in a 54 year old gentleman, which was successfully treated conservatively with a 6 week course of antibiotics.

Contributors: OSS: manuscript writing and literature review; $\mathrm{RN}$ : case management and manuscript review; TMV: transesophageal echocardiogram and critical review of manuscript; GV, ANK: manuscript review. OSS will act as guarantor. All authors approved the final version of manuscript.

Funding: None; Competing interests: None stated.

\section{References}

1. Ross D. Homograft replacement of the aortic valve. Lancet. 1962;2:487.

2. Yacoub M, Klieverik L, Melina G, Edwards S, Sarathchandra P, Bogers A. An evaluation of the Ross operation in adults. J Heart Valve Dis. 2006;15(4):531539.

3. Ashrafian H, Griselli M, Rubens MB, Mullen MJ, Sethia B. Pulmonary homograft endocarditis 19 years after a Ross procedure. Thorac Cardiovasc Surg. 2007;55(1):5556.

4. Gamez A, Castillo JC, Bonilla JL, Anguita MP. Infective endocarditis after the Ross procedure. Int $\mathrm{J}$ Cardiol. 2011;147(3):e53-54 .

5. Wong V, Cheng $\mathrm{CH}$, Chan KC. Fetal and neonatal outcome of exposure to anticoagulants during pregnancy. Am J Med Genet. 1993;45:17-21.

6. Chan WS, Anand S, Ginsberg JS. Anticoagulation of pregnant women with mechanical heart valves: a systematic review of the literature. Arch Intern Med. 2000;160:191-196.

7. Blome-Eberwein SA, Mrowiski D, Hpfmeister J, Hetzer R. Impact of mechanical heart valve prosthesis sound on patients quality of life. Ann Thorac Surg. 1996;61:594602. 